\title{
Fluoxetine plus cognitive behavioural therapy was most effective for adolescents with major depressive disorder
}

March J, Silva S, Petrycki S, et al; Treatment for Adolescents With Depression Study (TADS) Team. Fluoxetine, cognitive-behavioral therapy, and their combination for adolescents with depression: Treatment for Adolescents with Depression Study (TADS) randomized controlled trial. JAMA 2004;292:807-20.

\section{Q In adolescents with major depressive disorder (MDD), how do fluoxetine, cognitive behavioural therapy (CBT), and} their combination compare for effectiveness?

\section{METHODS}

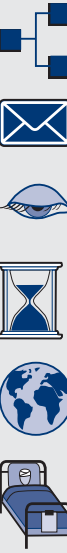

Design: randomised controlled trial (RCT).

Allocation: \{concealed\}.

Blinding: blinded (patients and healthcare providers [fluoxetine $v$ placebo comparison], and outcome assessors).

Follow up period: 12 weeks.

Setting: outpatient clinic in the US

Patients: 439 adolescents (mean age $15 y, 54 \%$ girls) who had a DSM-IV diagnosis of MDD, could attend an outpatient clinic, scored $\geqslant 45$ on the Children's Depression Rating Scale-Revised (CDRS-R), had a full scale $I Q \geqslant 80$, and were not taking antidepressants. Exclusion criteria included bipolar disorder, severe conduct disorder, substance abuse/dependence, 2 failed selective serotonin reuptake inhibitor (SSRI) trials, and poor response to $\mathrm{CBT}$

$\mathbf{R}_{\mathbf{X}}$ Intervention: fluoxetine, from $10 \mathrm{mg} /$ day to a maximum of 40 $\mathrm{mg} /$ day by week $8(\mathrm{n}=109) ; \mathrm{CBT}(\mathrm{n}=111)$; CBT plus fluoxetine $(n=107)$; or placebo $(n=112)$. CBT consisted of 15 sessions (5060 min each) for 12 weeks and aimed to correct depressive thought patterns and increase positive reinforcing behaviour patterns.

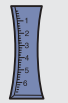

Outcomes: CDRS-R score (range 17-133; high scores = greater depression); Suicidal Ideation Questionnaire-Junior High School Version score; and Clinical Global ImpressionsImprovement (CGI-I) response rate (much improved or very much improved)

Patient follow up: $82 \%$ (100\% included in intention to treat analysis).

*Information provided by author.

\section{MAIN RESULTS}

Fluoxetine plus CBT led to greater improvement in CDRS-R scores than placebo $\left(\{\right.$ mean difference 7.6$\left.\},{ }^{*} \mathrm{p}=0.001\right)$, fluoxetine mean difference 4.4$\left.\},{ }^{*} \mathrm{p}=0.02\right)$, or CBT (\{mean difference 9.4\}, $\mathrm{p}=0.001)$. Fluoxetine plus CBT also reduced suicidal thoughts. Fluoxetine plus CBT and fluoxetine had greater CGI-I response rates relative to placebo and CBT alone. Numbers needed to treat were 3 (95\% CI 2 to 4) for fluoxetine plus CBT and 4 (CI 3 to 8 ) for fluoxetine alone relative to placebo. The 2 fluoxetine groups had more harm related events than the CBT alone and placebo groups (table)

For correspondence: $\mathrm{Dr} J \mathrm{~S}$ March, Department of Psychiatry, Duke University Medical Center, Durham, NC, USA. jsmarch@acpub.duke.edu Sources of funding: National Institute of Mental Health; fluoxetine and placebo supplied by Lilly Inc.

\section{CONCLUSION}

In adolescents with major depressive disorder, fluoxetine plus cognitive behavioural therapy was most effective.

${ }^{*}$ Calculated from data in article.

A modified version of this abstract appears in Evidence-Based Medicine.

Fluoxetine alone or fluoxetine plus cognitive behavioural therapy (CBT) $v$ CBT alone or placebo for major depressive disorder in adolescents*

\begin{tabular}{llll}
\hline Outcome & Event rates & RRI $(95 \% \mathrm{Cl})$ & NNH (CI) \\
\hline $\begin{array}{l}\text { Harm related adverse } \\
\text { events }\end{array}$ & $10 \%$ v 4.9\% & $107 \%$ (3 to 292) & 19 (7 to 713)
\end{tabular}

*Abbreviations defined in glossary; RRI, $\mathrm{NNH}$, and $\mathrm{Cl}$ calculated using odds ratio in article.

\section{Commentary}

ew well designed studies have assessed treatments for MDD in adolescents. A recent public health advisory from the US Food and Drug Administration (FDA) ${ }^{1}$ questioned the safety of SSRIs because of increased suicidal behaviours in adolescents and children. ${ }^{2}$ In the UK, the regulator has strongly advised against the use of all SSRIs except fluoxetine in adolescents and children (http://www.mhra.gov.uk/news/ ssri_101203.htm accessed 13 Jan 2005).

TADS offers some strong support for 12 weeks of fluoxetine plus $\geqslant 11$ sessions of simultaneous CBT. Study strengths are the RCT design, double blinding in the fluoxetine and placebo groups, the inclusion of adolescents with comorbidities, and the data safety and monitoring protocol conducted by the National Institute of Mental Health.

Patients in the CBT plus fluoxetine and CBT alone groups were not blinded, which might have affected the findings if these patients expected improved outcomes. Adolescents with substance abuse, recent suicide attempts, or suicidal ideation were excluded; thus findings are not generalisable to all adolescents with MDD. Some of these adolescents are most in need of treatment. A study weakness was the exclusion of nonEnglish speaking adolescents or parents. The modal income reflected a high middle class, thus missing many adolescents with lower socioeconomic status who have MDD. The study report did not describe how the fidelity of the CBT intervention was monitored. The treatment duration of 12 weeks does not offer support for longer use of either treatment.

TADS provides evidence that fluoxetine plus CBT is effective for adolescents with $M D D$, including those with some comorbid conditions. However, the advisory from the FDA should also be considered. Antidepressant medications may increase the risk of suicidality or other harm related adverse events. Nurses must continue to monitor depressed adolescents for suicidal thinking and behaviour. This includes providing anticipatory guidance to parents or guardians about signs to observe and actions to take. Further study is needed to determine the longer term effects of combined pharmacological and psychotherapeutic treatments for the wide array of adolescents who have MDD.

Lynn Rew, RN, EdD, FAAN University of Texas at Austin, Austin, Texas, USA

1 US Food and Drug Administration, Center for Drug Evaluation and Research. Suicidality in children and adolescents being treated with antidepressant medications. http://www.fda.gov/cder/drug/ antidepressants/SSRIPHA200410.htm (accessed 12 Jan 2005).

2 Medicines and Healthcare Products Regulatory Agency. http:// www.mhra.gov.uk/news/ssri_101203.htm (accessed 12 Jan 2005). 